15. Jensen NA, Baron Van Evercooren A, Mattei MG, et al. Neurological disorder in transgenic mice that express the large $\mathrm{T}$ antigen of polyoma virus in the nervous system. Mol Biol Med 1989 ; 6 : 493-500.

16. Griep AE, Westphal H. Differentiation versus proliferation of transgenic mouse lens cells expressing polyoma large $T$ antigen : evidence for regulation by an endogenous growth factor. New' Biologist $1990 ; 2$ : 727-38.

17. Sinn E, Muller WV, Pattengale P, Tepler $I$, Wallace $R$, Leder $P$. Coexpression of MMTV/Ha-ras and MMTV/c-my'c genes in transgenic mice : synergistic action of oncogenes in vivo. Cell 1987; 49 : 465-75.

18. Dademans W, Perraud F, Le Meur M, Gerlinger P, Courtney M, Pavirani A. Heterologous protein expression by trans immortalized differentiated liver cell lines derived from transgenic mice (hepatomad $\alpha-1$-antitrypsin/ONC mouse). Biologicals 1990) ; $18: 191-8$.

19. Jallat S, Perraud F, Dalemans W, et al. Characterization of recombinant factor IX expressed in transgenic mice and in clerived trans-immortalized hepatic cell lines. EMBO J $1990 ; 9$ : 3295-301

20. Sifers RN, Rogers BB, Hawkins HK, Finegold MJ, Woo SLC. Elevated synthesis of human $\alpha-1$-antitrypsin hinders the secretion of murine $\alpha$-1-antitrypsin from hepatocytes of transgenic mice. J Biol Chem 1989 ; 264: 15696-700.

21. Mac Kay K, Striker LJ, Elliot S, Pin kert CA, Brinster RL, Striker GE. Glomerular epithelial, mesanglial and endothelial cell lines from transgenic mice. Kidney Int 1988 ; $33: 677-84$

22. Efrat S, Teitelman G, Anwar M, Ruggiero D, Hanahan D. Glucagon gene regulatory region directs oncoprotein expression to neurons and pancreatic $\alpha$ cells. Neuron 1988 ; 1: 605-13.

23. Behringer RR, Peschon JJ, Messing A, et al. Heart and bone tumors in transgenic mice. Proc Natl Acad Sci USA 1988; 85 : 2648-52.

24. Spanopoulou E, Early A, Elliot J, et al. Complex lymphoid and epithelial thymic tumors in Thy 1-myc: transgenic mice. Nature 1989 ; 342 : 185-9.

25. Alexander WS, Schrader JW, Adams JM. Expression of the c-myc oncogene under control of an immunoglobulin enhancer in E $\mu$-myc transgenic mice. Mol Cell Biol $1987 ; 1436-44$

26. Leder A, Pattengale PK, Kuo A, Stewart TA, Leder P. Consequences of widespread deregulation of the c-myc gene in transgenic mice : multiple neoplasms. Cell $1986 ; 45: 485-95$.

27. Nakamura $T$, Mahon DA, Miskin $R$, Dey A, Kuwabara T, Westphasl H. Differentiation and oncogenesis: phenotypically distinct lens tumors in transgenic mice. New Biologisl 1989 ; 1 : 193-204.

28. Sippola-Thiele M, Hanahan D, Howley PM. Cell-heritable stages of tumor progression in transgenic mice harboring the bovine papillomavirus type 1 genome. Mol Cell Biol 1989 ; 9 : 925-34.

29. Williams RL, Courtneidge SA, Wagner EF. Embryonic lethadities and endothelial tumors in chimeric mice expressing polyoma virus middle $\mathrm{T}$ oncogene. Cell $1988 ; 52$ : 121-31.

\title{
Transfert de gènes dans les cellules nerveuses. Immortalisation cellulaire et marquage génétique
}

\author{
Claudine Évrard, Marc Le Bert, Isabelle Borde, Éric Galiana, \\ Rozenn Bernard, Pierre Rouget
}

$\mathbf{L}$ a compréhension des mécanismes cellulaires et moléculaires intervenant dans la différenciation, le contrôle de la prolifération et les interactions des cellules nerveuses est encore grandement limitée par la difficulté d'établir des lignées cellulaires stables (donc un matériel clonal,

C. Évrard: maîlre de conférences à l'universilé Paris VII. M. Le Bert, I. Borde, R. Bernard : étudiants en cours de thèse. E. Galiana : stagiaire post-docloral. P. Rouget : professeur à l'universilé Paris VI. Laboratoire de biologie moléculaire et différenciation, biochimie cellulaire, université Paris VI et Collège de France, 11, place Marcelin-Berthelot, 75231 Paris Cedex 05, France.

homogène, reproductible et quantitativement illimité), variées et conservant l'essentiel des propriétés des cellules de départ. En effet, la plupart des lignées de cellules nerveuses étudiées jusqu'à récemment étaient tumorigènes et avaient perdu le contrôle de leur division et de leur différenciation. Cependant, quelques lignées issues de tumeurs [1-4], d'autres obtenues in vitro après transformation spontanée ou virale [5-7], ont déjà permis de clarifier certains aspects de la neurogenèse.

Des perspectives nouvelles ont été ouvertes par les travaux des laboratoires de F. Cuzin [8] et R. Weinberg [9]. Sur des fibroblastes murins, ces auteurs ont montré que la transformation oncogène nécessite une coopération entre différents gènes et implique plusieurs étapes dont la première, appelée immortalisation, confère à ces cellules un potentiel de division illimité, sans qu'elles acquièrent un phénotype transformé ou tumoral. A l'issue de cette première étape qui peut être induite par l'expression des séquences grand $\mathrm{T}$ du virus polyome, E1A d'adénovirus et certains gènes de la famille $m y c$, la prolifération cellulaire reste contrôlée par inhibition de contact, les fibroblastes ne se multiplient pas en absence d'ancrage et ne deviennent pas tumorigènes. A la suite de ces observations, il est devenu envisa- 
geable d'établir des lignées permanentes de cellules ayant conservé l'essentiel du contrôle de leur prolifération et de leur différenciation.

Transfert d'oncogènes dans les cellules nerveuses et établissement de lignées immortalisées

Nous avons ainsi été incités à initier un nouveau système, fondé sur le transfert et l'intégration d'oncogènes définis, permettant l'immortalisation des différentes catégories de cellules nerveuses murines [10] et vraisemblablement extrapolable à d'autres types cellulaires. Les gènes immortalisants grand $T$ du virus polyome, E1A d'adénovirus et parfois le gène grand $T$ de SV40 ont été introduits à l'aide de différentes méthodes (transfection, électroporation, lipofection, transduction à l'aide de vecteurs rétroviraux recombinants) dans des cellules issues de régions déterminées du cerveau. Ce travail a conduit à l'établissement de lignées permanentes non phénotypiquement transformées, correspondant à diverses sous-populations de cellules nerveuses et précurseurs mixtes, dont quelques-unes ont déjà été décrites [10-12]. Avec une démarche analogue, quelques autres laboratoires ont immortalisé des précurseurs glioneuronaux dont l'étude de la différenciation in vitro a confirmé l'existence de progéniteurs communs aux cellules gliales et neuronales $[13,14]$. Toutefois, dans tous les cas, la fréquence d'obtention de lignées immortalisées capables d'effectuer leur différenciation terminale in vitro, demeure faible. En effet, les gènes transférés sont intégrés préférentiellement dans les cellules ayant conservé un potentiel de division important, ce qui confère aux précurseurs précoces un avantage sélectif visà-vis de l'immortalisation. Afin d'augmenter l'efficacité des vecteurs et de cibler l'expression des oncogènes, nous avons placé ces gènes sous le contrôle de promoteurs neuro-spécifiques (région promotrice du gène neurofilaments- $L$ ), ou glio-spécifiques (régions régulatrices du gène GFAP ou du papovavirus JC). à une autre approche : à partir de souris transgéniques portant le gène grand $T$ du virus polyome, construites par M. Rassoulzadegan et F. Cuzin, nous avons établi différentes lignées immortalisées de cellules nerveuses, issues de régions définies du cerveau [15]. Parallèlement, deux autres laboratoires ont obtenu des lignées transformées à partir de tumeurs provenant d'animaux transgéniques portant les séquences grand $\mathrm{T}$ de SV40, placées sous contrôle de promoteurs neuro-spécifiques [16, 17].

\section{Différenciation in vitro des différen- tes lignées de cellules nerveuses immortalisées}

Les lignées immortalisées à l'aide de ces diverses méthodes peuvent être schématiquement réparties en différentes catégories :

Certaines présentent des caractéristiques d'astrocytes de type 1 . Elles expriment les formes $120 \mathrm{kDa}$ et $140 \mathrm{kDa}$ des molécules d'adhérence cellulaire de type N-CAM, synthétisent la laminine durant leur phase de multiplication puis la sécrètent lorsque leur croissance est inhibée par contact. Elles expriment la glial fibrillary acidic protein (GFAP, spécifique des astrocytes) après confluence et cette expression est corrélée à l'arrêt de la prolifération. Certaines de ces lignées présentent des récepteurs à divers neuromédiateurs couplés à la phospholipase $\mathrm{C}$ : par exemple, $\alpha 1$-adrénergiques, muscariniques et sérotoninergiques [15].

Chez d'autres lignées, précurseurs d'astrocytes de type 1, l'expression de la GFAP est inductible par la FGF [10]. Quelques-unes, correspondant probablement à des précurseurs plus précoces, expriment N-CAM et laminine, sont capables de stimuler le développement neuritique, mais ne sont pas inductibles pour l'expression de GFAP.

Une autre famille de lignées [11] présente des propriétés de précurseurs mixtes oligodendrocyte-astrocytes de type 2 et une grande analogie avec les cellules O-2A mises en évidence par M. Raff au niveau du nerf optique [18]. Au cours de leur croissance, elles synthétisent d'abord les gangliosides reconnus par l'anticorps A2B5 qui interagit avec divers types de précurseurs gliaux [18] ou neuronaux [19]. Ensuite, selon les conditions de culture, elles expriment soit le galactocérébroside, spécifique de cellules oligodendrogliales, soit la glutamine synthétase et la GFAP.

D'autres lignées présentent des caractéristiques de précurseurs mixtes astrocyte-neurones [12-14]. Leur différenciation dans l'une ou l'autre direction dépend aussi des conditions de culture : ces cellules expriment, par exemple, soit la GFAP, soit les diverses formes de neurofilaments spécifiques des neurones. Certaines de ces lignées, issues du striatum, expriment des récepteurs à divers neuromédiateurs [12] : récepteurs $\alpha 1$-adrénergiques, muscariniques, glutamatergiques, sérotoninergiques de la sousclasse 5-HT2, couplés positivement à la phospholipase $\mathrm{C}$; récepteurs $\beta$ adrénergiques, D1 et D2 dopaminergiques, couplés soit positivement, soit négativement à l'adénylate cyclase. Nous étudions actuellement, d'une part, l'expression de ces récepteurs au cours de la différenciation, d'autre part, l'effet des neuromédiateurs correspondants sur la prolifération et la différenciation cellulaire. Une collaboration avec le laboratoire de $\mathrm{J}$. Mallet a conduit à transférer le gène de la tyrosine hydroxylase ( $\mathrm{TH}$, enzyme clé de la biosynthèse de dopamine) dans ces lignées. Un objectif de ce travail est d'examiner les régulations coordonnées entre l'expression du gène $T H$, celle des récepteurs dopaminergiques et des protéines $G$ intervenant dans la transduction des signaux. Une autre perspective est d'améliorer la réparation de lésions striatales après greffe de ces cellules chez l'animal [20].

Transfert et intégration du gène lac $Z$ : marquage génétique

C. Cepko et ses collaborateurs avaient montré que la transduction du gène lac $Z$ à l'aide de vecteurs rétroviraux, dans les cellules du système nerveux central, permettait l'étude des filiations cellulaires [21]. En effet, l'expression $\mathrm{m} / \mathrm{s} n^{\circ} 4 \mathrm{vol} .7$, auril 91 
de ce gène, qui code pour la $\beta$ galactosidase, peut être suivie histochimiquement, in situ, par l'hydrolyse du substrat chromophore X-Gal. Bien que la détection simultanée de certains marqueurs de différenciation soit possible [11], l'association du gène lac $Z$ à un signal de localisation nucléaire (nls $[22,23])$ facilite la caractérisation des cellules et lève toute ambiguïté due à l'expression éventuelle d'une galactosidase endogène [24]. Afin d'obtenir une expression ciblée des séquences nls-lacZ dans des neurones ou des astrocytes, ou encore des oligodendrocytes, nous avons placé ces séquences sous le contrôle des régions promotrices des gènes neurofilaments ou $G F A P$, ou encore des régions régulatrices du papovavirus JC. Ce travail a maintenant permis d'obtenir différents types de lignées, correspondant à divers précurseurs et catégorics cellulaires du système nerveux central, à la fois immortalisées, ayant intégré et exprimant les séquences lac $Z$, y compris après leur différenciation terminale. L'expression stable de la $\beta$ galactosidase, soit dans les cellules des lignées, soit dans des cellules issues de culture primaire ou encore de souris transgéniques portant les séquences nls-lacZ, doit permettre d'étudier in vitro et également in vivo, après greffe des cellules marquées, les interactions cellulaires directes, le contrôle de la prolifération et de la différenciation de ces cellules.

\section{Quelques perspectives}

Compte tenu des progrès déjà effectués ou attendus dans le domaine du transfert et de l'intégration des gènes dans les cellules eucaryotes, il est raisonnable de penser que, dans un proche avenir, de nombreuses lignées cellulaires d'origine et de stade de différenciation variés pourront être obtenues. En tant que matériel cellulaire homogène, de telles lignées devraient, par exemple, permettre d'étudier avec une plus grande précision la synthèse de neuromédiateurs, de neuropeptides, de facteurs de croissance et de différenciation, ainsi que l'expression des récepteurs correspondants et la transduction des signaux.

Des améliorations dériveront certainement d'une meilleure compréhension de l'oncogenèse, du rôle des oncogènes et antioncogènes, de leurs interactions et de leur équilibre dans un tissu ou une catégorie cellulaire précise.

D'autres progrès concernent la construction de vecteurs. Ainsi, le tropisme relativement étroit des vecteurs rétroviraux vis-à-vis des espèces animales peut maintenant être élargi en modifiant les glycoprotéines de l'enveloppe virale. Par ailleurs, les vecteurs porteurs d'oncogènes dont l'activité est conditionnelle ou dont l'expression est modulable, peuvent permettre de mieux contrôler l'induction de la différenciation en arrêtant à volonté la division cellulaire. Toutefois, les vectcurs inductibles utilisés actuellement ne sont pas totalement satisfaisants; par exemple, dans le cas des vecteurs dont l'expression est dépendante des glucocorticoïdes, l'inducteur peut avoir également un effet direct sur la différenciation ; ceux impliquant la région promotrice du gène codant pour la métallothionéine ne sont que faiblement inductibles. Bien que les mutants thermosensibles soient d'un grand intérêt dans l'étude des mécanismes de la transformation, leur utilisation en vue de l'obtention de lignées cellulaires est souvent limitée par un échappement des effets de ces mutations. Cependant, une des lignées transformées à l'aide de ce type de mutants s'est avérée intéressante car elle est capable d'exprimer des marqueurs neuronaux à température non permissive [14].

Le fait de focaliser l'expression des gènes transférés en choisissant des promoteurs appropriés devrait faciliter l'établissement de lignées à partir de populations cellulaires bien déterminées. De plus, la possibilité d'intégrer, en un site précis du génome, les gènes transférés a été ouverte récemment par la mise au point de systèmes conduisant à des recombinaisons homologues [25]. L'ensemble de ces progrès concernant les constructions de vecteurs eucaryotes et les méthodes de transfert de gènes contribueront certainement à renforcer et à préciser les approches de thérapie génique

\section{Summary}

Gene transfer into neural cells. Immortalization and genetic labelling of the cells

Early sequences from polyoma virus, SV40, and adenovirus were transferred into murine primary cells from different brain areas, using several methods. Different types of immortalized and phenotypically untransformed cell lines were obtained, including various astroglial lines, oligodendrocyteastrocyte common progenitors, bipotential neurone-astrocyte precursors that were able to express several neurotransmitter receptors. Another approach was the establishment of permanent cell lines from transgenic mice carrying the polyoma large $T$ gene. In order to visualize these cells, even when placed in interaction with other cells, the lac $Z$ gene was integrated in the genome of some lines.

\section{Références}

1. Schubert D, Heineman S, Carlisle W, et al. Clonal cell lines from the rat central nervous system. Nalure 1974 ; 249 : 224-7.

2. Greene LA, Tischler AS. Establishment of a noradrenergic clonal line of rat adrenal pheochromocytoma cells which respond to nerve growth factor. Proc Nall Acad Sci USA $1976 ; 73$ : 2424-8.

3. Benda P. Rodent glial cells. In : Schoffeniels E, Franck G, Towers DB, Hertz L, eds. Dynamic Properties of Glia Cells. Oxford: Pergamon Press, 1978 : 67-81.

4. Ronnet GV, Hester LD, Nye JS, Connors K, Snyder SH. Human cortical neuronal cell line : establishment from a patient with unilateral megalencephaly. Science 1990 ; 248: 603-5.

5. Alliot F, Pessac B. Astrocytic cell clones derived from established cultures of 8 dayspostnatal mouse cerebella. Brain Res 1984 ; 306: 283-97

6. De Vitry F. Growth and differentiation of a primitive nervous cell line after in vivo transplantation into syngeneic mice. Nalure 1977 ; 267 : 48-50.

7. Mallat M, Mouro-Neto U, Gros F, Glowinski J, Prochiantz A. Two simian virus 40 (SV40)-transformed cell lines from the mouse striatum and mesencephalon presenting astrocytic characters. II. Interactions with mesencephalic neurons. Dev Brain Res 1986 $26: 23-33$ 
8. Rassoulzadegan M, Naghashfar Z, Cowie A, et al. Expression of individual polyoma virus early proteins in oncogenic transformation. Proc Natl Acad Sci USA 1983: 80 4354-8.

9. Land H, Parada LF, Weinberg RA Tumorigenic conversion of primary embryo fibroblasts requires at least two cooperating oncogenes. Nature 1983; 304: 596-602.

10. Evrard C, Galiana E, Rouget P. Establishment of " normal " nervous cell lines after transfer of polyoma virus and adenovirus early genes into murine brain cells. EMBOJ 1986 ; 5: 3157-62.

11. Évrard C, Galiana E, Rouget P. Immortalization of bipotential glial progenitors and generation of permanent "bluel " cell lines. $J$ Neurosci Res 1988 ; 21 : 80-7.

12. Évrard C, Borde I, Marin P, et al. Immortalization of bipotential and plastic glioneuronal precursor cells. Proc Natl Acad Si USA $1990 ; 87: 3062-6$

13. Bartlett PF, Reid HH, Bailey KA, Bernard $\mathrm{O}$. Immortalization of mouse neural precursor cells by c-myc oncogene. Proc Natl Acad Sci USA 1988 ; 85 : 3255-9.

14. Frederiksen K, Jat PS, Valtz N, Levy
D, McKay R. Immortalization of precursor cells from the mammalian CNS. Neuron $1988 ; 1$ : 439-48.

15. Galiana E, Borde I, Marin P, et al. Establishment of permanent astroglial cell lines, able to differentiate in vitro, from transgenic mice carrying the polyoma virus large $T$ gene an alternative approach to brain cell immortalization. J Neurosci Res $1990 ; 27$ : 269-77. 16. Hammang JP, Baetge EE, Behringer RR, Brinster RL, Palmiter RD, Messing A. Immortalized retinal neurons derived from SV40 T-antigen-induced tumors in transgenic mice. Neuron $1990 ; 4$ : 775-82.

17. Mellon PL, Windle JJ, Goldsmith PC, Padula CA, Roberts JL, Weiner RI. Immortalization of hypothalamic GnRH neurons by genetically targeted tumorigenesis. Neuron $1990 ; 5: 1-10$

18. Raff MC, Miller RH, Noble M. A glial progenitor cell that develops in vitro into an astrocyte or an oligodendrocyte depending on culture medium. Nature $1983 ; 303: 390-6$. 19. Eisenbarth GS, Walsh FS, Nirenberg M. Monoclonal antibody to a plasma membrane antigen of neurons. Proc Natl Acad Sci USA $1979 ; 76: 4913-7$.
20. Horellou P, Brundin P, Kalen P, Mallet J, Björklund A. In vivo release of DOPA and dopamine from genetically engineered cells grafted to the denervated rat striatum. Neuron 1990 ; 5 : 393-402.

21. Price J, Turner D, Cepko C. Lineage analysis in the vertebrate nervous system by retrovirus-mediated gene transfer. Proc Natl Acad Sci USA 1987 ; 84: 156-60.

22. Kalderon D, Roberts BL, Richardson WD, Smith AE, A short amino acid sequence able to specify nuclear location. Cell 1984; 39 : 499-509.

23. Bonnerot C, Rocancourt D, Briand P, Grimber G, Nicolas JF. A $\beta$-galactosidase hybrid protein targeted to nuclei as a marker for developmental studies. Proc Natl Acad Sci USA 1987; 84 : 6795-9.

24. Galileo DS, Gray GE, Owens GC, Majors J, Sanes JR. Neurons and glia arise from a common progenitor in chicken optic tectum : demonstration with two retroviruses and cell type-specific antibodies. Proc Natl Acad Sci USA 1990; 87 : 458-62.

25. Capecchi MR. Altering the genome by homologous recombination. Science 1989; 244: 1288-92.

\title{
Immortalisation de cellules germinales mâles de souris transgéniques par l'antigène grand $T$ du virus polyome
}

\author{
M. Rassoulzadegan, V. Paquis, F. Vidal, R. Loubière, F. Cuzin \\ TEXTE NON REÇU
}

\section{Résumé}

Une série de 44 familles transgéniques a été obtenue après microinjection du plasmide pPyLT1, codant pour la protéine grand $\mathrm{T}$ du virus polyome. Les mâles de toutes les familles expriment l'ARN viral et l'antigène $T$ dans les testicules. L'hybridation in situ localise l'expression du transgène à l'intérieur des tubes séminifères, dans les zones

M. Rassoulzadegan, V. Paquis, F. Vidal, R. Loubière, F. Cuzin : Inserm U. 273, Nice, France. pré et post-méiotiques. Ces mâles demeurent asymptomatiques pendant la plus grande partie de leur vie, mais tous sans exception, entre 15 mois et deux ans, développent une forme unique de cancer bilatéral des testicules. Le tableau pathologique ne correspond pas à l'un des cancers classiques de ces organes (lymphome, séminome, carcinome embryonnaire, etc.). Ces cellules se divisent efficacement en culture, mais elles ne sont pas transplantables dans l'animal. Maintenues en croissance, elles montrent une morphologie homogène sans différenciation évidente. Dans des conditions défavorables pour la croissance (maintien prolongé à confluence, carence en sérum), on note l'apparition de deux types morphologiques distincts, des cellules de grande taille avec des noyaux lobés caractéristiques et, associées à celles-ci, des petites cellules à noyaux extrêmement compacts. Ces deux morphologies évoquent, respectivement, des cellules de Sertoli et des spermatides. Nous cherchons actuellement à optimaliser les conditions de culture pour cette différenciation et à la caractériser au niveau moléculaire (expression de gènes spécifiques de la lignée germinale mâle). 